\title{
LOW 5-STARS AT 5-VERTICES IN 3-POLYTOPES WITH MINIMUM DEGREE 5 AND NO VERTICES OF DEGREE FROM 7 TO $9^{1}$
}

\author{
Oleg V. Borodin, Mikhail A. Bykov \\ AND \\ Anna O. Ivanova \\ Sobolev Institute of Mathematics \\ Novosibirsk, 630090, Russia \\ e-mail: brdnoleg@math.nsc.ru \\ 131093@mail.ru \\ shmgnanna@mail.ru
}

\begin{abstract}
In 1940, Lebesgue gave an approximate description of the neighborhoods of 5-vertices in the class $\mathbf{P}_{\mathbf{5}}$ of 3-polytopes with minimum degree 5 .

Given a 3-polytope $P$, by $h_{5}(P)$ we denote the minimum of the maximum degrees (height) of the neighborhoods of 5 -vertices (minor 5 -stars) in $P$.

Recently, Borodin, Ivanova and Jensen showed that if a polytope $P$ in $\mathbf{P}_{5}$ is allowed to have a 5 -vertex adjacent to two 5 -vertices and two more vertices of degree at most 6 , called a $(5,5,6,6, \infty)$-vertex, then $h_{5}(P)$ can be arbitrarily large. Therefore, we consider the subclass $\mathbf{P}_{\mathbf{5}}^{*}$ of 3 -polytopes in $\mathbf{P}_{\mathbf{5}}$ that avoid $(5,5,6,6, \infty)$-vertices.

For each $P^{*}$ in $\mathbf{P}_{\mathbf{5}}^{*}$ without vertices of degree from 7 to 9 , it follows from Lebesgue's Theorem that $h_{5}\left(P^{*}\right) \leq 17$. Recently, this bound was lowered by Borodin, Ivanova, and Kazak to the sharp bound $h_{5}\left(P^{*}\right) \leq 15$ assuming the absence of vertices of degree from 7 to 11 in $P^{*}$.

In this note, we extend the bound $h_{5}\left(P^{*}\right) \leq 15$ to all $P^{*}$ s without vertices of degree from 7 to 9 .
\end{abstract}

Keywords: planar map, planar graph, 3-polytope, structural properties, 5-star, weight, height.

2010 Mathematics Subject Classification: 05C75.

\footnotetext{
${ }^{1}$ The work was funded by the Russian Science Foundation, grant 16-11-10054.
} 


\section{REFERENCES}

[1] O.V. Borodin, Structural properties of planar maps with the minimal degree 5, Math. Nachr. 158 (1992) 109-117. doi:10.1002/mana.19921580108

[2] O.V. Borodin and A.O. Ivanova, Describing 4-stars at 5-vertices in normal plane maps with minimum degree 5, Discrete Math. 313 (2013) 1710-1714. doi:10.1016/j.disc.2013.04.025

[3] O.V. Borodin and A.O. Ivanova, Light neighborhoods of 5-vertices in 3-polytopes with minimum degree 5, Sib. Èlektron. Mat. Izv. 13 (2016) 584-591. doi:10.17377/semi.2016.13.045

[4] O.V. Borodin and A.O. Ivanova, Light and low 5-stars in normal plane maps with minimum degree 5, Sib. Math. J. 57 (2016) 470-475. doi:10.1134/S0037446616030071

[5] O.V. Borodin, A.O. Ivanova and T.R. Jensen, 5-stars of low weight in normal plane maps with minimum degree 5, Discuss. Math. Graph Theory 34 (2014) 539-546. doi:10.7151/dmgt.1748

[6] O.V. Borodin, A.O. Ivanova and O.N. Kazak, Describing neighborhoods of 5-vertices in 3-polytopes with minimum degree 5 and without vertices of degrees from 7 to 11 , Discuss. Math. Graph Theory 38 (2018) 615-625. doi:10.7151/dmgt.2024

[7] O.V. Borodin, A.O. Ivanova, O.N. Kazak and E.I. Vasil'eva, Heights of minor 5-stars in 3-polytopes with minimum degree 5 and no vertices of degree 6 and 7, Discrete Math. 341 (2018) 825-829. doi:10.1016/j.disc.2017.11.021

[8] O.V. Borodin, A.O. Ivanova and D.V. Nikiforov, Low minor 5-stars in 3-polytopes with minimum degree 5 and no 6-vertices, Discrete Math. 340 (2017) 1612-1616. doi:10.1016/j.disc.2017.03.002

[9] O.V. Borodin, A.O. Ivanova and D.V. Nikiforov, Low and light 5-stars in 3-polytopes with minimum degree 5 and restrictions on the degrees of major vertices, Sib. Math. J. 58 (2017) 600-605. doi:10.1134/S003744661704005X

[10] O.V. Borodin and D.R. Woodall, Short cycles of low weight in normal plane maps with minimum degree 5, Discuss. Math. Graph Theory 18 (1998) 159-164. doi:10.7151/dmgt.1071

[11] P. Franklin, The four color problem, Amer. J. Math. 44 (1922) 225-236. doi: $10.2307 / 2370527$

[12] S. Jendrol' and T. Madaras, On light subgraphs in plane graphs of minimum degree five, Discuss. Math. Graph Theory 16 (1996) 207-217. doi:10.7151/dmgt.1035

[13] H. Lebesgue, Quelques conséquences simples de la formule d'Euler, J. Math. Pures Appl. 19 (9) (1940) 27-43. 
[14] E. Steinitz, Polyeder und Raumeinteilungen, in: Enzykl. Math. Wiss. (Geometrie), 3 (1922) 1-139.

[15] P. Wernicke, Über den kartographischen Vierfarbensatz, Math. Ann. 58 (1904) 413-426.

doi:10.1007/BF01444968

Received 18 December 2017

Revised 25 June 2018

Accepted 25 June 2018 